\title{
Human milk induces fetal small intestinal cell proliferation - involvement of a different tyrosine kinase signaling pathway from epidermal growth factor receptor
}

\author{
Takashi Takeda, Masahiro Sakata, Ryoko Minekawa, Toshiya \\ Yamamoto, Masami Hayashi, Keiichi Tasaka and Yuji Murata \\ Division of Obstetrics and Gynecology, Department of Specific Organ Regulation, Osaka University Graduate School of Medicine, 2-2 Yamadaoka, Suita, \\ Osaka 565-0871 Japan \\ (Requests for offprints should be addressed to Takashi Takeda; Email: take@gyne.med. osaka-u.ac.jp)
}

\begin{abstract}
Breast milk has non-nutritional protective effects on recipient infants. It has been speculated that bioactive substances present in human milk have important roles in protecting infants. However, the mechanisms by which such substances protect newborns are unclear. Therefore, we analyzed the growth-promoting activity of human milk and the intracellular signaling mechanism thereof using human fetal small intestinal (FHS 74 Int) cells. Epidermal growth factor (EGF) stimulated the proliferation of these cells. However, this stimulation was less effective than that of aqueous milk (5\% vol $/ \mathrm{vol})$. The bioactivity of human milk was heat stable but protease sensitive. EGF receptor tyrosine kinase inhibitor did not repress the milk-induced growth-promoting effect on fetal small intestinal cells. Regarding the intracellular signaling pathway, the milkinduced cell proliferation pathway was tyrosine kinase
\end{abstract}

dependent but was neither mitogen-activated protein (MAP) kinase nor phosphatidylinositol-3 (PI-3) kinase dependent. On the other hand, EGF-induced cell proliferation was tyrosine kinase, MAP kinase, and PI-3 kinase dependent. Rapid tyrosine phosphorylation of several intracellular proteins was detected after milk stimulation. Furthermore, the time course of phosphorylation induced by milk was different from that induced by EGF. The sizes of the proteins phosphorylated in response to milk were different from those of the Shc proteins phosphorylated in response to EGF. These results suggest that human milk induces fetal intestinal cell proliferation through a unique tyrosine kinase pathway different from the EGF receptor signaling pathway.

Journal of Endocrinology (2004) 181, 449-457

\section{Introduction}

Breast milk has been shown to have many benefits for infants, including not only a reduced risk of neonatal necrotizing enterocolitis, gastroenteritis, respiratory infection and immunologically based disease but also improved later cognitive development (Wagner et al. 1996, Lucas et al. 1998, Schanler \& Atkinson 1999). These clinical findings indicate that milk may exert protective effects on infants during neonatal maturation. It is well known that milk contains a variety of proteins, peptides, and steroids that possess biological activity (Grosvenor et al. 1993). It is speculated that these bioactive substances play important roles in the non-nutritional effects of human milk.

The newborn gastrointestinal tract undergoes maturational changes in the weeks after birth. Human milk is known to stimulate gastrointestinal mucosal proliferation and maturation in animal models and is thought to protect the neonatal infant from harmful environmental factors by affecting the mucosal barrier (Wagner et al.
1996). Some of the growth factors in human milk, such as epidermal growth factor (EGF), transforming growth factor alpha (TGF $\alpha$ ) and insulin-like growth factors (IGFs), have been shown to stimulate the proliferation of intestinal cells and the formation of the mucosal barrier (Corps \& Brown 1987, Ichiba et al. 1992, Wagner et al. 1998, Wagner \& Forsythe 2000). Among them, EGF is thought to be the main stimulator in human milk (Grosvenor et al. 1993).

Numerous studies have been performed to investigate the intracellular signaling pathways of many growth factors and cytokines in a variety of cells in the past decade. Both EGF and TGF $\alpha$ bind to the same EGF receptor (EGFR). Ligands binding to EGFR activate the receptor tyrosine kinase activity, resulting in both autophosphorylation and phosphorylation of various intracellular effector proteins (Moghal \& Sternberg 1999). Shc is an adapter molecule that links receptor tyrosine kinase to downstream signaling pathways. The activated EGFR recruits and phosphorylates Shc and subsequently activates 
the Ras-mitogen-activated protein (MAP) kinase pathway. The activated EGFR also tyrosine phosphorylates p85, a phosphatidylinositol-3 kinase (PI-3 kinase) subunit, and increases PI-3 kinase activity (Moghal \& Sternberg 1999). IGFs (IGF-I and IGF-II) bind to the IGF-I receptor (IGF-IR). The activated IGF-IR activates both the Ras-MAP kinase pathway and the PI-3 kinase pathway (Blakesley et al. 1996).

However, the proliferative effects of such growth factors on human small intestinal cells are thought to account only partially for the growth promotion by human milk (Ichiba et al. 1992, Wagner et al. 1998), and the signal transduction pathway by which human milk stimulates intestinal cell proliferation remains unknown. Therefore, in this study we analyzed the mechanism by which milk induces human fetal intestinal cell proliferation.

\section{Materials and Methods}

Growth factors, cytokine, antibodies and kinase inhibitor

Human recombinant EGF and insulin were purchased from Sigma-Aldrich (St Louis, MO, USA). The antibodies used were anti-phosphotyrosine monoclonal antibody (PY20, Santa Cruz Biotechnology, Inc., Santa Cruz, CA, USA), anti-phosphotyrosine-EGFR polyclonal antibody (Tyr1173, Santa Cruz Biotechnology) and anti-Shc polyclonal antibody (Upstate Biotechnology, Lake Placid, NY, USA) for Western blotting. The specific inhibitor of the tyrosine kinase activity of EGF receptor (AG1478, SigmaAldrich), tyrosine kinase inhibitor (genistein, SigmaAldrich), JAK-2 tyrosine kinase inhibitor (AG490, Sigma-Aldrich), PI-3 kinase inhibitor (wortmannin, Sigma-Aldrich) and MEK inhibitor (PD-98059, SigmaAldrich) were dissolved in DMSO, and $1 \mu$ of these stock solutions $(1 \% \mathrm{vol} / \mathrm{vol})$ was added to the culture medium at the concentrations indicated in the Results section. Control cells were treated with $1 \mu \mathrm{l}$ DMSO ( $1 \% \mathrm{vol} / \mathrm{vol})$ to control for the cytotoxic effect of DMSO itself.

\section{Cells and cell culture}

A human fetal small intestinal cell line, FHs 74 Int (ATCC CCL241), was purchased from Health Science Research Resources Bank (Osaka, Japan) and was cultured in DMEM (Gibco BRL, Life Technologies, Inc., Grand Island, NY, USA) containing 10\% fetal calf serum (FCS; CSL, Parkville, Victoria, Australia), EGF (30 ng/ml) and insulin $(10 \mu \mathrm{g} / \mathrm{ml})$ as described previously (Wagner et al. 1998).

\section{Milk samples}

Six samples of human mammary secretions from six mothers of full-term infants were obtained 2 to 4 days after parturition. Subjects donated milk samples after giving written informed consent. The samples were stored at $4{ }^{\circ} \mathrm{C}$ and processed within $12 \mathrm{~h}$ of expression and then pooled. Milk was centrifuged for $20 \mathrm{~min}$ at $1000 \mathrm{~g}$ to remove fat and cellular debris as described previously (Smith-Kirwin et al. 1998). The aqueous layer was stored at $-20^{\circ} \mathrm{C}$ until it was assayed. To deplete the steroid hormones, aqueous human milk was treated with dextran-coated charcoal (Sigma-Aldrich) as described previously (Provost et al. 2000). To inactivate heat-sensitive proteins and peptides in milk, aqueous milk was boiled for $5 \mathrm{~min}$ and then centrifuged for $20 \mathrm{~min}$ at $1000 \mathrm{~g}$. The aqueous layer obtained thereby was used for experiments. Heat inactivation of EGF was carried out in the same way as heat inactivation of milk. For protease digestion, human milk was incubated at $50{ }^{\circ} \mathrm{C}$ for $24 \mathrm{~h}$ with $1.5 \mathrm{mg} / \mathrm{ml}$ predigested pronase (Roche Molecular Biochemicals, Mannheim, Germany) as described previously (Pimental et al. 1996). To concentrate and desalt aqueous milk, the sample was centrifuged using a Microcon Centrifugal Filter Device (YM-100, which could retain the molecules above $100 \mathrm{kDa}$ ) (Nihon Millipore, Tokyo, Japan) and reconstituted in PBS.

\section{Cell proliferation assay}

Cell proliferation was measured using the MTS (3(4,5-dimethylthiazol-2-yl)-5-(3-carboxymethoxyphenyl)-2 (4-sulfophenyl)-2H-tetrazolium) assay (CellTiter 96 AQueous One Solution Cell Proliferation Assay, Promega, Madison, WI, USA). Cells were placed in complete medium (10\% FCS with EGF and insulin) at a concentration of $5 \times 10^{3}$ cells/well in 96-well plates for $24 \mathrm{~h}$. Subsequently, the cells were incubated in $100 \mu \mathrm{E}$ EGF and insulin-free DMEM with FCS and bovine serum albumin ( $1 \mathrm{mg} / \mathrm{ml}$, Sigma-Aldrich) for another $24 \mathrm{~h}$. Milk samples or growth factors were added at the concentrations indicated in the Results section and the cells were further cultured for $24 \mathrm{~h}$. Then the MTS assay was performed as recommended by the manufacturer. All experiments were performed in quadruplicate and repeated at least three times with similar results. Results are expressed as the change in cell proliferation. The average values of data from a representative experiment are presented with standard deviations indicated by bars. Statistical analysis was performed by the unpaired $t$-test. Statistical significance was set at $P<0 \cdot 05$.

\section{Immunoprecipitation, SDS-PAGE and Western blotting}

Cells were harvested and lysed for $60 \mathrm{~min}$ in $1 \mathrm{ml}$ lysis buffer (50 mM Tris (pH 7.5), $150 \mathrm{mM} \mathrm{NaCl}, 1 \mathrm{mM}$ EDTA, $0 \cdot 5 \%$ NP-40, $1 \mathrm{mM}$ sodium orthovanadate, $1 \mathrm{mM}$ $\mathrm{NaF}, 0.75 \mathrm{mM}$ phenylmethylsulfonyl fluoride, $15 \%$ glycerol, and $10 \mu \mathrm{g} / \mathrm{ml}$ each of aprotinin, pepstatin, and leupeptin) as described (Lutticken et al. 1994). Proteins in 
the lysates from $2 \times 10^{5}$ cells were immunoprecipitated with the anti-phosphotyrosine monoclonal antibody. Immune complexes were separated by SDS-PAGE $(7 \cdot 5 \%)$, and transferred to an Immobilon-P nylon membrane (Nihon Millipore), which was then immunoblotted with the relevant primary antibody as described (Takeda et al. 1994). Proteins were detected by enhanced chemiluminescence (ECL; Amersham Biosciences Corp. Piscataway, NJ, USA) and rehybridization was performed as described (Takeda et al. 1994).

\section{Results}

Human milk and EGF stimulate the proliferation of fetal intestinal cells

We tested the growth-promoting activity of milk and EGF on human fetal intestinal cells by using the MTS assay (Fig. 1A and B). Both of these factors could induce cell proliferation dose-dependently, but EGF was not as effective as milk. As the concentration of EGF in breast milk has been reported to be $30-50 \mathrm{ng} / \mathrm{ml}$ (Ichiba et al. 1992), the final concentration of this growth factor in 5\% (vol/ vol) milk medium was calculated as $1.5-2.5 \mathrm{ng} / \mathrm{ml}$. In previous reports, it was demonstrated that a plateau in the stimulation of cell proliferation by EGF was reached at the 3-300 ng/ml concentration range (Ichiba et al. 1992, Wagner et al. 1998). The concentrations of EGF used in these experiments $(0 \cdot 5-500 \mathrm{ng} / \mathrm{ml})$ were thus sufficient to induce observable effects and were much higher than those in $5 \%$ breast milk. These facts indicate that the growth-promoting activity of milk is not due solely to the effect of EGF. Although it has been reported that in the case of fibroblast proliferation EGF is a major growthpromoting agent in human milk (Carpenter 1980), our data suggest that there is some other bioactive substance in the case of intestinal cell proliferation. In the following experiments we characterized the growth-promoting agent in milk.

Growth-promoting activity of milk is sensitive to pronase digestion, but not to charcoal treatment and heat inactivation

It has been reported that steroid hormones, including estradiol, estriol, progesterone and other steroid hormones, are present in human milk (Grosvenor et al. 1993). We examined whether removing steroids from milk by charcoal treatment affects the growth-promoting activity of milk for intestinal cells (Fig. 2A). Stripping the steroids from milk did not affect the growth-promoting activity, suggesting that steroid hormones are not the major growth-promoting factors in human milk.

Next we analyzed the effect of heat inactivation of milk and EGF on their growth-promoting activity (Fig. 2B). Boiled milk could activate cell proliferation as effectively as
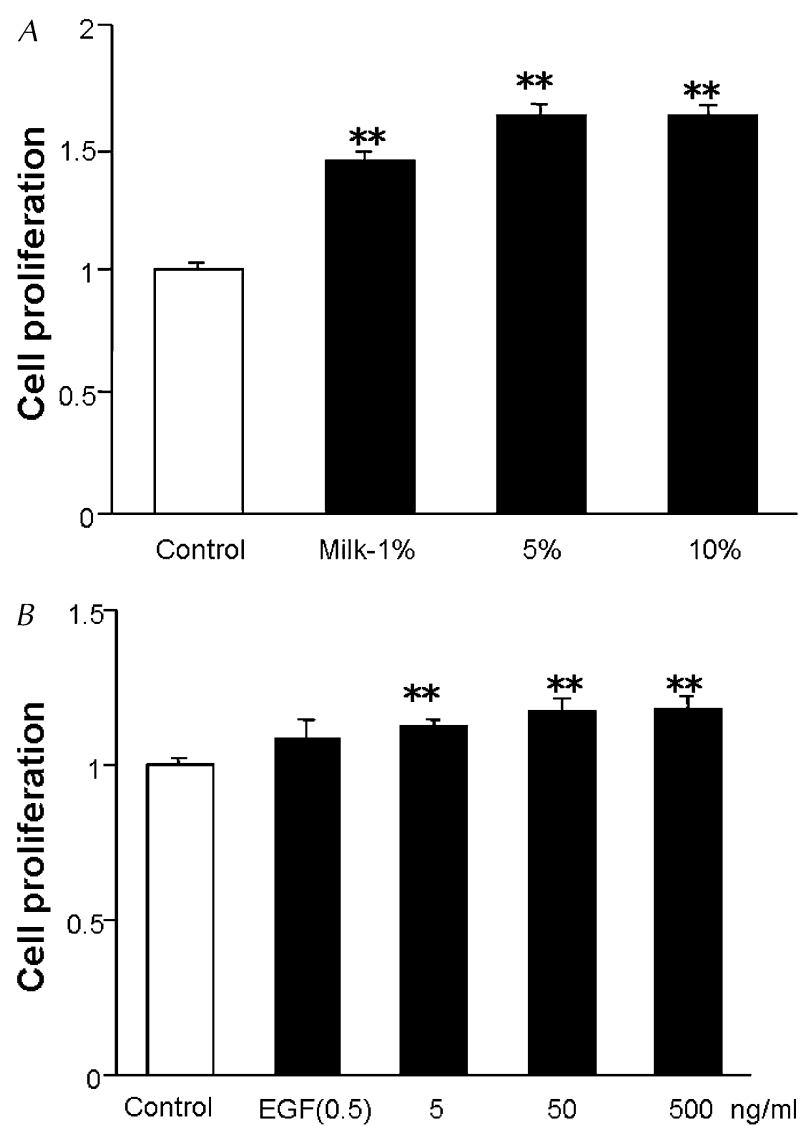

Figure 1 Growth-promoting activity of human milk and EGF. FHs 74 Int cells were treated with (A) human milk (1-10\% vol/vol), or (B) EGF $(0 \cdot 5-500 \mathrm{ng} / \mathrm{ml})$ for $24 \mathrm{~h}$. Cell proliferation was measured using the MTS assay, in which the relative cell proliferation was estimated by measuring the change in optical density, measured by spectrophotometry at $490 \mathrm{~nm}$, and expressed relative to that in the control cells taken as 1 . The values are averages of quadruplicates of a representative experiment; more than three independent experiments were carried out with similar results. ${ }^{* *} P<0 \cdot 01$ versus control.

non-boiled milk. In contrast, boiled EGF could not activate cell proliferation at all. This result shows that the growthpromoting activity of milk is heat stable, suggesting the possibility that this bioactive factor is not a protein or a peptide. To investigate this possibility, we digested milk with pronase at $50{ }^{\circ} \mathrm{C}$ (Fig. 2C). Pronase digestion abolished the growth-promoting activity of milk. However, incubation of milk at $50{ }^{\circ} \mathrm{C}$ for $24 \mathrm{~h}$ without pronase did not affect the growth-promoting activity. These results show that the growth-promoting factors in milk are heatstable proteins or peptides. To study whether the growthpromoting effects of milk were due to components other than proteins, we concentrated the protein fraction of milk and reconstituted it in PBS. The exchange of buffer and the depletion of small molecule substances did not change 

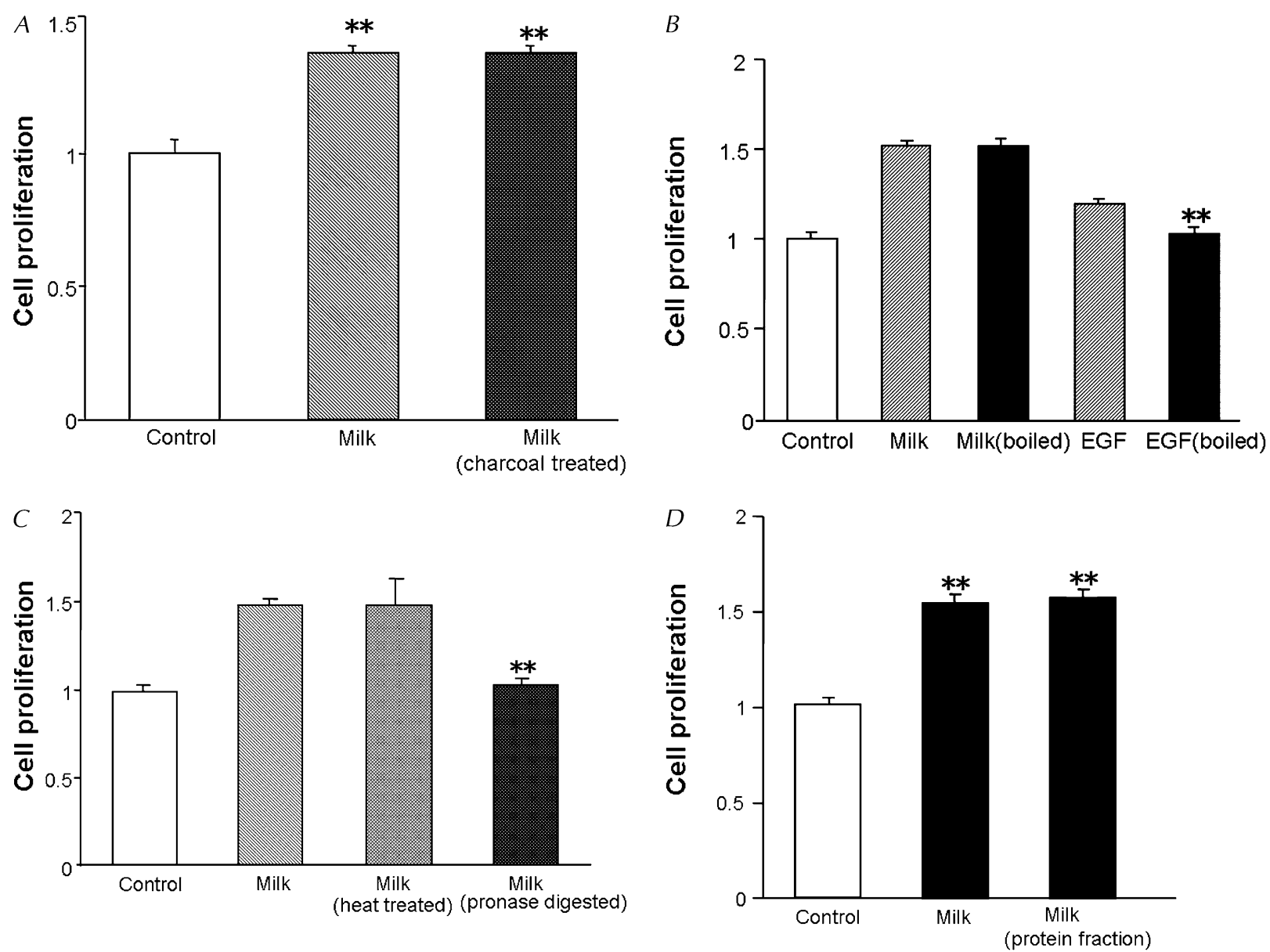

Figure 2 The growth-promoting factor in milk is a heat-stable protein or peptide. (A) Steroid-stripping of milk did not impair the growth-promoting activity. FHs 74 Int cells were treated with human milk (5\% vol/vol) or charcoal-treated milk ( $5 \% \mathrm{vol} / \mathrm{vol})$ for $24 \mathrm{~h}$. Steroid-stripping of milk by charcoal treatment was carried out as described in Materials and Methods. ${ }^{* *} P<0 \cdot 01$ versus control. No significant difference was observed between Milk and Milk (charcoal treated) groups. (B) The growth-promoting activity in milk is heat stable. FHs 74 Int cells were treated with human milk (5\% vol/vol), heat-inactivated milk (Milk (boiled), 5\% vol/vol), EGF (50 ng/ml) and heatinactivated EGF (EGF (boiled), $50 \mathrm{ng} / \mathrm{ml}$ ) for $24 \mathrm{~h}$. Heat inactivation of milk and EGF by boiling were carried out as described in Materials and Methods. No significant difference was observed between Milk and Milk (boiled) groups. ${ }^{* *} P<0 \cdot 01$, EGF versus EGF (boiled). (C) The growth-promoting activity in milk is pronase sensitive. FHs 74 Int cells were treated with pronase-containing buffer (control, 5\% vol/vol), human milk in buffer (Milk, 5\% vol/vol), human milk diluted in buffer and incubated at $50{ }^{\circ} \mathrm{C}$ for $24 \mathrm{~h}$ (Milk (heat treated), $5 \% \mathrm{vol} / \mathrm{vol}$ ) or human milk digested with pronase (Milk (pronase digested), $5 \% \mathrm{vol} / \mathrm{vol}$ ) for $24 \mathrm{~h}$. Pronase digestion of milk was carried out as described in Materials and Methods. No significant difference was observed between Milk and Milk (heat treated). ${ }^{* *} P<0 \cdot 01$, Milk (pronase digested) versus human milk (Milk). (D) The growth-promoting factor in milk is located in the protein fraction. FHs 74 Int cells were treated with human milk $(5 \% \mathrm{vol} / \mathrm{vol})$ or the protein fraction of milk ( $5 \% \mathrm{vol} / \mathrm{vol})$ for $24 \mathrm{~h}$. The protein fraction of milk was concentrated with an ultrafiltration device as described in Materials and Methods. ${ }^{* *} P<0 \cdot 01$ versus control. No significant difference was observed between Milk and Milk (protein fraction) groups. Cell proliferation was measured using the MTS assay and expressed relative to that of the control cells taken as 1 . Values were calculated as described in the legend to Fig. 1. The values are averages of quadruplicates of a representative experiment; more than three independent experiments were carried out with similar results.

the growth-promoting activity (Fig. 2D). This result indicates that bioactive substances other than proteins in milk do not affect the proliferation of intestinal cells.

\section{EGFR-mediated signaling is not the major pathway of milk-induced intestinal cell proliferation}

We analyzed the effect of a specific inhibitor of the tyrosine kinase activity of EGFR (AG1478) on the milk-induced growth-promoting activity (Fig. 3). AG1478 inhibited the EGF-induced cell proliferation effectively, but did not impair the growth-promoting activity of milk. This result indicates that EGFR-mediated signaling is not the major pathway of milk-induced intestinal cell proliferation. As shown in Fig. 3, milkinduced cell proliferation rates seemed to be higher than those seen in Figs 1 and 2. In this experiment we treated control cells with medium containing 1\% DMSO. The 


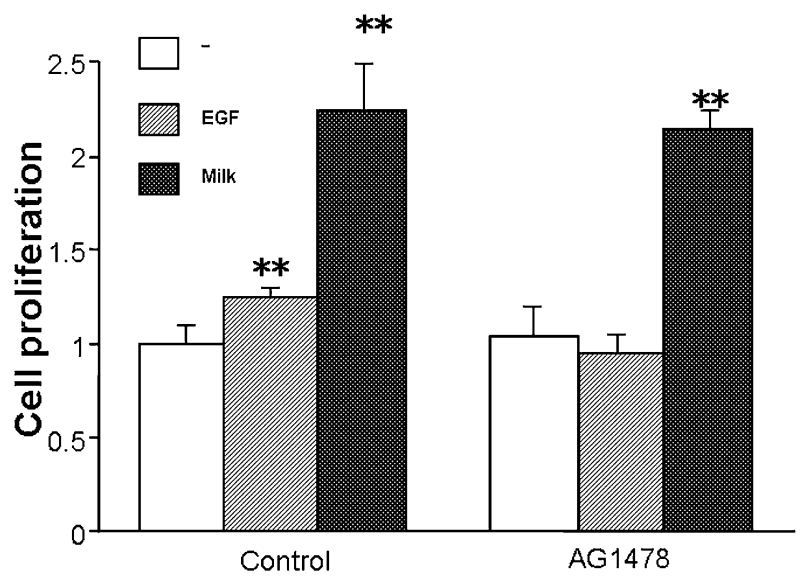

Figure 3 EGFR-mediated signaling is not the major pathway in milk-induced intestinal cell proliferation. An inhibitor of the tyrosine kinase activity of EGFR (AG1478) did not repress the growth-promoting activity of milk. FHs 74 Int cells were treated with EGF $(50 \mathrm{ng} / \mathrm{ml})$ or with human milk $(5 \% \mathrm{vol} / \mathrm{vol})$ with or without AG1478 $(2 \cdot 0 \mu \mathrm{M})$ for $24 \mathrm{~h}$. Control cells were treated with DMSO ( $1 \% \mathrm{vol} / \mathrm{vol})$. Cell proliferation was measured using the MTS assay and expressed relative to that of the non-stimulated control cells $(-)$ taken as 1 . Values were calculated as described in the legend to Fig. 1. The values are averages of quadruplicates of a representative experiment; more than three independent experiments were carried out with similar results. ${ }^{* *} P<0.01$ versus control cells $(-)$.

cell proliferation of the 1\% DMSO-treated cells was about $80-90 \%$ that of untreated cells and this cytotoxic effect of DMSO was completely abrogated by treatment with $5 \%$ milk (data not shown). Accordingly, the milk-induced cell proliferation rates with medium containing $1 \%$ DMSO are higher than those without DMSO.

Milk-induced intestinal cell proliferation depends on a tyrosine kinase pathway, but not on the MAP kinase or PI-3 kinase pathway

Many growth factors act via intracellular signaling pathways that involve protein tyrosine kinases (PTKs). There are two major signaling pathways that involve PTKs, the MAP kinase and PI-3 kinase pathways (Moghal \& Sternberg 1999). We studied the effects of a PTK inhibitor (genistein), a MEK inhibitor (PD-98059) and a PI-3 kinase inhibitor (wortmannin) on milk- or EGF-induced intestinal cell proliferation (Fig. 4A and B). Genistein repressed the growth-promoting activity of milk, but PD-98059 and wortmannin did not. On the other hand genistein and PD-98059 repressed the growth-promoting activity of EGF completely and wortmannin repressed it partially. These results indicate that milk-induced intestinal cell proliferation depends on a PTK pathway, but not the MAP kinase or the PI-3 kinase pathway, and that
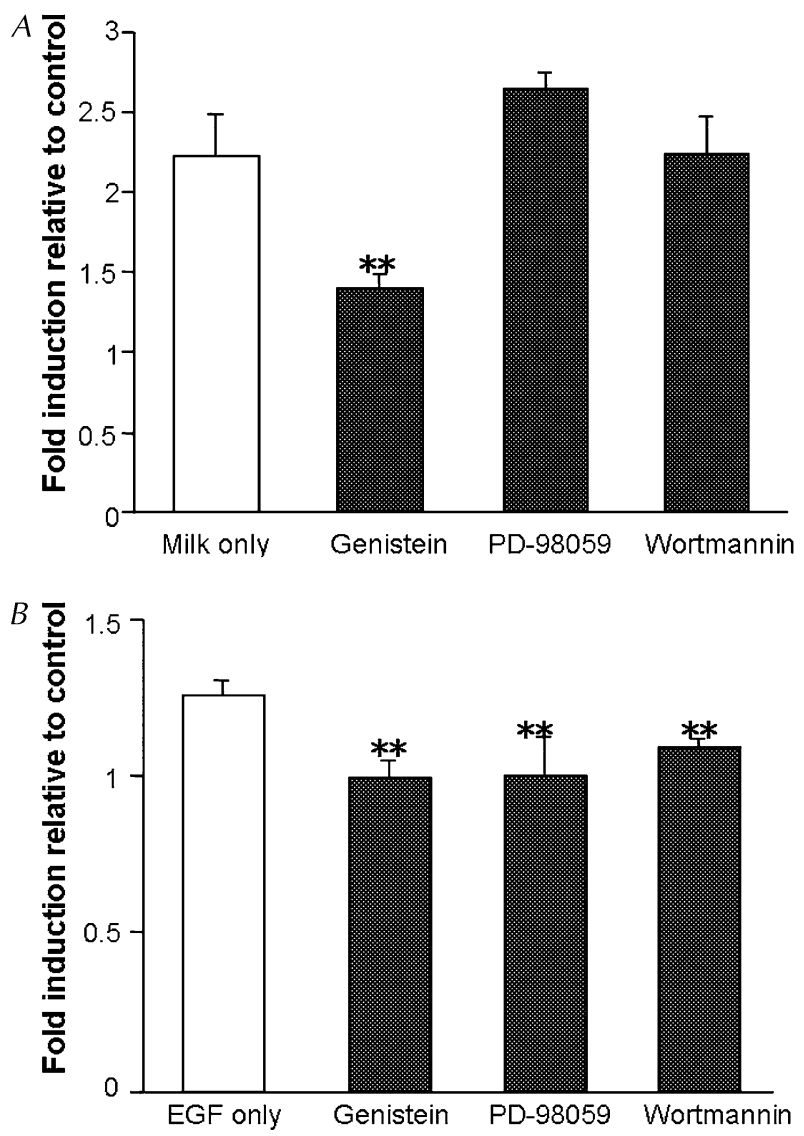

Figure 4 Milk-induced intestinal cell proliferation depends on a PTK pathway, but not on the MAP kinase or PI-3 kinase pathway. FHs 74 Int cells were treated with (A) human milk ( $5 \% \mathrm{vol} / \mathrm{vol})$ or (B) EGF $(50 \mathrm{ng} / \mathrm{ml}$ ) with or without PTK inhibitor (genistein, $25 \mu \mathrm{g} / \mathrm{ml}$ ), MAP kinase inhibitor (PD-98059, $50 \mu \mathrm{M}$ ), or Pl-3 kinase inhibitor (wortmannin, $1000 \mathrm{nM}$ ) for $24 \mathrm{~h}$. Control cells were treated with DMSO ( $1 \% \mathrm{vol} / \mathrm{vol})$. Cell proliferation was measured using the MTS assay and growth-promoting activity of milk was expressed as fold induction. Fold induction was calculated as the ratio of proliferation of cells treated with milk (A) or EGF $(B)$ to that of cells incubated with DMSO (1\% vol/vol). The values are averages of quadruplicates of a representative experiment; more than three independent experiments were carried out with similar results. ${ }^{* *} P<0 \cdot 01$ versus Milk only in (A) and EGF only in (B).

the EGF-induced signal transduction pathway is different from the milk-induced pathway.

\section{$J A K$ kinase pathway has a repressive effect on the growth-promoting activity of milk}

Studies on the signal transduction of interferons have shown that a novel tyrosine kinase, JAK kinase, plays a major role in signal transduction for a variety of cytokines and hormones (Hirano 1998). EGF and IGFs are also 


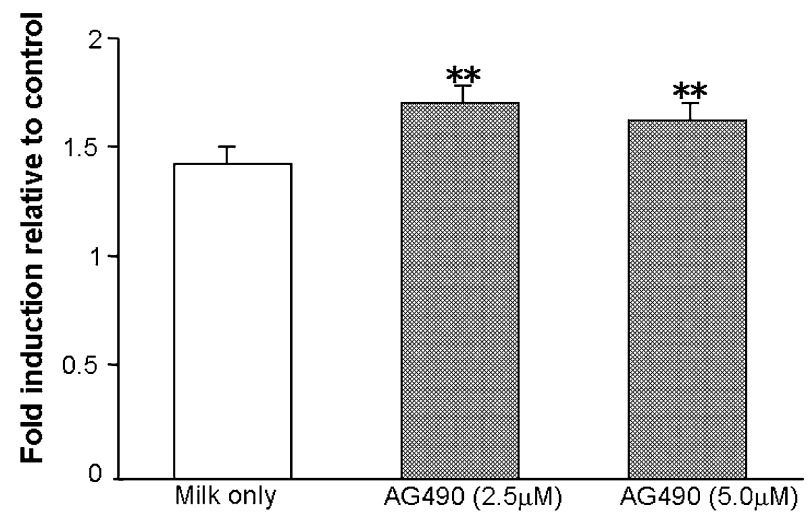

Figure 5 Repressive effect of the JAK kinase pathway on the growth-promoting activity of milk. FHs 74 Int cells were treated with human milk $(5 \% \mathrm{vol} / \mathrm{vol})$ with or without the JAK kinase inhibitor (AG490, $2.5 \mu \mathrm{M}$ or $5 \mu \mathrm{M}$ ) for $24 \mathrm{~h}$. Control cells were treated with DMSO ( $1 \% \mathrm{vol} / \mathrm{vol})$. Cell proliferation was measured using the MTS assay and the growth-promoting activity of milk was expressed as fold induction. Fold induction was calculated as the ratio of proliferation of cells treated with milk to that of cells incubated without milk. The values are averages of quadruplicates of a representative experiment; more than three independent experiments were carried out with similar results. ${ }^{* *} P<0 \cdot 01$ versus Milk only.

known to activate the JAK kinase pathway in addition to the MAP kinase pathway and the PI-3 kinase pathway (Zhong et al. 1994, Zong et al. 2000). We examined whether JAK kinase is involved in milk-induced intestinal cell proliferation (Fig. 5). JAK-2 tyrosine kinase inhibitor (AG490) increased the growth-promoting activity of milk. This indicates that the JAK kinase pathway has a repressive effect on the growth-promoting activity of milk.

Milk induces rapid tyrosine phosphorylation by activating a pathway different from that induced by EGF

We analyzed whether milk could induce tyrosine phosphorylation of cellular proteins (Fig. 6A upper panel). At least two proteins ranging in size from $46 \mathrm{kDa}$ to $66 \mathrm{kDa}$ were rapidly tyrosine phosphorylated in cells treated with milk. The peak of phosphorylation occurred at $15 \mathrm{~min}$ and the peak level of phosphorylation was sixfold higher than that in control cells. Next, we analyzed the EGF-induced tyrosine phosphorylation (Fig. 6B upper panel). The molecular sizes of the rapidly phosphorylated proteins seemed to be the same as those of the milk-induced proteins, but the peak of phosphorylation occurred at 5 min. The peak level of EGF-induced phosphorylation was only 1.2-fold higher than that in control cells (Fig. 6B upper panel). As the sizes of these phosphorylated proteins resembled those of Shc isoforms (46, 52 and $66 \mathrm{kDa})$, we reprobed the same membrane with anti-Shc antibody after stripping (Fig. 6A and B lower panels). The sizes of these proteins were different from those of the proteins whose tyrosine phosphorylation was induced by milk. The peak protein levels occurred at $5 \mathrm{~min}$ in EGF-stimulated cells, but the protein levels were not changed in milk-stimulated cells.

Next, we studied the effects of a PTK inhibitor (genistein), an EGFR tyrosine kinase inhibitor (AG1478), a MEK inhibitor (PD-98059), a PI-3 kinase inhibitor (wortmannin) and a JAK kinase inhibitor (AG490) on milk or EGF-induced tyrosine phosphorylation (Fig. 6C). Genistein repressed the milk and EGF-induced phosphorylation, but AG1478 repressed only EGF-induced phosphorylation. These results indicate that the milkinduced tyrosine phosphorylation pathway is different from the EGFR-mediated signaling pathway. Other kinase inhibitors, PD-98059, wortmannin and AG490 did not repress the milk- or EGF-induced phosphorylation. These results indicate that the milk- and EGF-induced tyrosine phosphorylation pathways are not downstream of MEK kinase, PI-3 kinase or JAK kinase. As the EGFinduced cell proliferation was very weak (Fig. 1B), it is possible that these cells have no EGFR and EGF activates another growth factor receptor. We further confirmed that EGFR was tyrosine phosphorylated in EGF-treated cells, but not in milk-treated cells by reprobing the same membrane with anti-phosphotyrosine-EGFR antibody after stripping (Fig. 6D). EGF tyrosine phosphorylated EGFR effectively, and genistein and AG1478 repressed this phosphorylation completely (Fig. 6D lower panel). On the other hand, we could not detect the tyrosine phosphorylated EGFR in milk-treated cells (Fig. 6D upper panel).

\section{Discussion}

This is the first report about the intracellular events involved in milk-induced intestinal cell proliferation. In this work we have shown that (1) EGFR-mediated signaling is not the major pathway in milk-induced intestinal cell proliferation, (2) the growth-promoting factor in milk is a heat-stable protein or peptide, (3) milk-induced intestinal cell proliferation depends on a PTK pathway, not the MAP kinase or PI-3 kinase pathway, (4) the JAK kinase pathway has a repressive effect on the growth-promoting activity of milk, and (5) milk induces rapid tyrosine phosphorylation by activating a pathway different from that induced by EGF.

In previous reports, the growth-promoting activities of EGF, TGF $\alpha$, IGF-I and insulin were examined using the same intestinal cells (FHs 74 Int cells) (Ichiba et al. 1992, Wagner et al. 1998, Wagner \& Forsythe 2000). As a much higher concentration of IGF-I than that present in milk was necessary to observe the growth-promoting activity of IGF-I (Wagner \& Forsythe 2000), IGF-I-induced cell proliferation seems not to be a physiological phenomenon. Although human colostrum contains high concentrations 


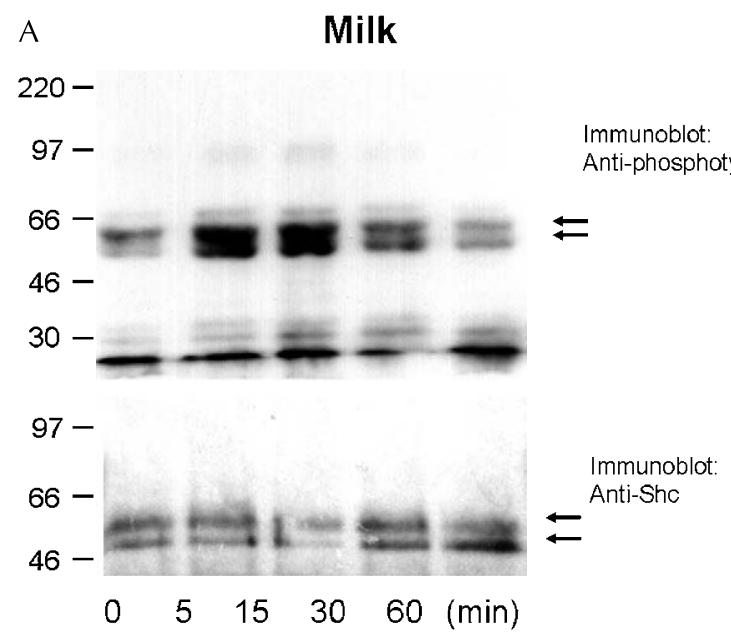

B EGF

$220-$

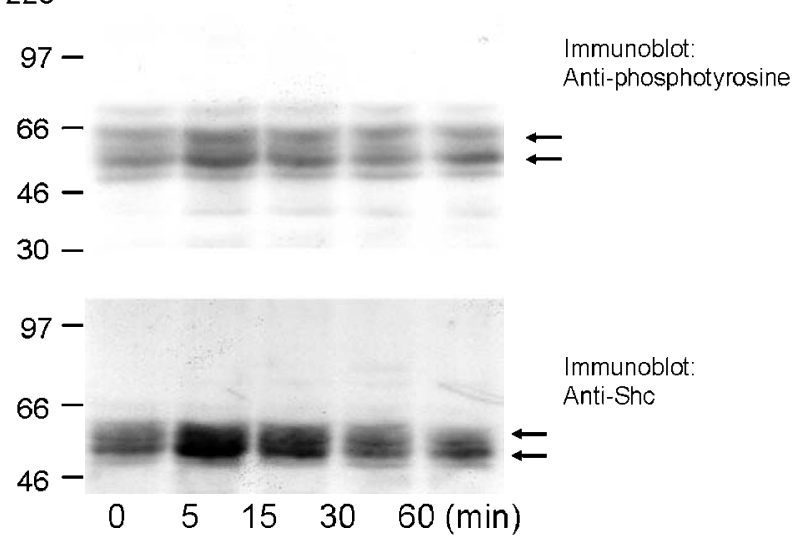

D Immunoblot: Anti-p-EGFR

C Immunoblot: Anti-phosphotyrosine

Milk
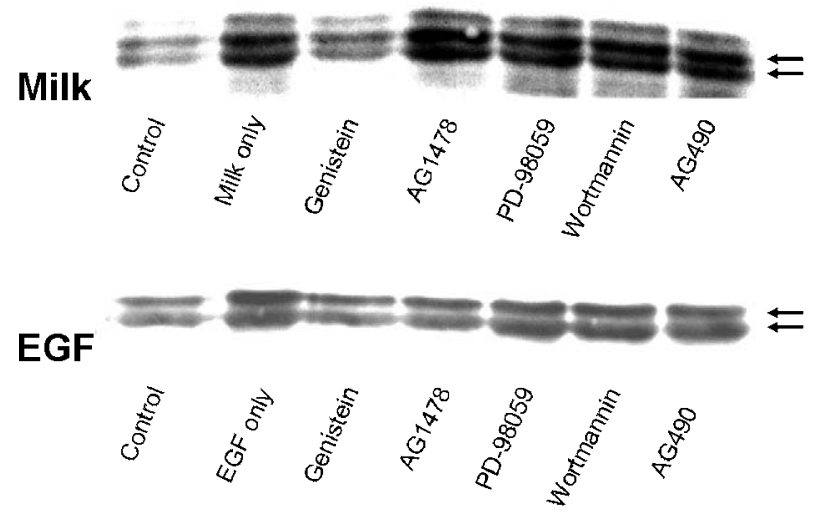

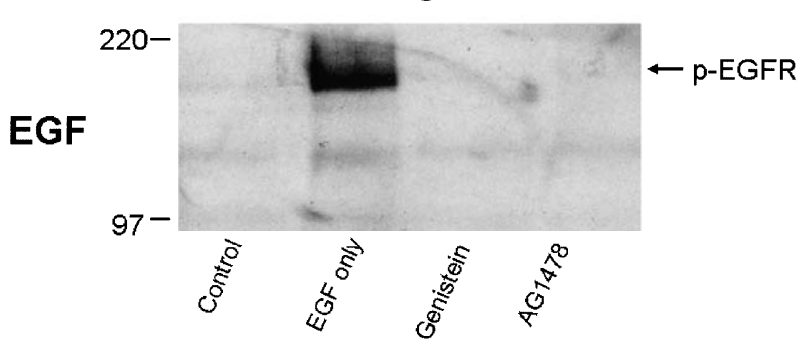

Figure 6 Milk induces rapid tyrosine phosphorylation by activating a pathway different from that induced by EGF. FHs 74 Int cells were treated for the indicated times with (A) boiled milk ( $5 \% \mathrm{vol} / \mathrm{vol})$ or (B) EGF $(50 \mathrm{ng} / \mathrm{ml})$. Cell lysates were immunoprecipitated with anti-phosphotyrosine antibody. Immune complexes were separated by SDS-PAGE and blotted with anti-phosphotyrosine antibody (A, B upper panels). The blots were then stripped and reprobed with anti-Shc antibody (A, B lower panels). The two arrows in A and B (upper panels) indicate the tyrosine-phosphorylated proteins induced by milk or EGF. The two arrows in A and B (lower panels) indicate Shc proteins (p46 and p52). (C) Milk-induced tyrosine phosphorylation depends on the PTK pathway, not on the EGFR tyrosine kinase, MAP kinase, PI-3 kinase or JAK kinase pathways. FHs 74 Int cells were pretreated with PTK inhibitor (genistein, $25 \mu \mathrm{g} / \mathrm{ml}$ ), EGFR tyrosine kinase inhibitor (AG1478, 2.0 $\mu \mathrm{M}$ ), MAP kinase inhibitor (PD-98059, $50 \mu \mathrm{M}$ ), Pl-3 kinase inhibitor (wortmannin, $1000 \mathrm{nM}$ ) or JAK kinase inhibitor (AG490, $2.5 \mu \mathrm{M}$ ) for $1 \mathrm{~h}$. Control cells were treated with DMSO (1\% vol/vol). Then the cells were treated with boiled milk $(5 \% \mathrm{vol} / \mathrm{vol})(\mathrm{C}$, upper panel) or with EGF $(50 \mathrm{ng} / \mathrm{ml})(\mathrm{C}$, lower panel) for $15 \mathrm{~min}$. Cell lysates were immunoprecipitated with anti-phosphotyrosine antibody. Immune complexes were separated by SDS-PAGE and blotted with anti-phosphotyrosine antibody. The blots were then stripped and reprobed with anti-phosphotyrosine-EGFR antibody (anti-p-EGFR) (D).

of insulin (3.75 $\pm 0.88 \mathrm{nM})$ (Read et al.1984), FHs 74 Int cells are not responsive to insulin in the range of concentrations found in milk (Ichiba et al. 1992). On the other hand, EGF can activate cell proliferation at a concentration expected in human milk $(3 \mathrm{ng} / \mathrm{ml})$, but its growthpromoting activity was weaker than that of human milk (Wagner \& Forsythe 2000). These previously reported results and our data are in accord (Fig. 1). It is possible that
FHs 74 Int cells have no EGFR or EGFR is not activated efficiently by EGF stimulation. Our data showed that EGF could tyrosine phosphorylate EGFR effectively (Fig. 6D lower panel).

Our data also showed that a PTK-dependent pathway stimulated by milk plays an important role in intestinal cell proliferation (Figs 4 and 6). PTK-dependent pathways also play roles in the signal transduction of other regulators of 
cell proliferation. Many growth factors (such as EGF, TGF $\alpha$ and IGF-I) use the Ras-MAP kinase pathway and the PI-3 kinase pathway, which are known signaling molecules downstream of PTK, but these pathways seem not to be important in milk-induced cell proliferation (Fig. 4). JAK family tyrosine kinases have been shown to function as central non-receptor tyrosine kinases in a pathway that has been characterized as a not-Ras-MAP kinase and a not-PI-3 kinase pathway (Hirano 1998). Therefore, we expected that this pathway might be involved in milk-induced cell proliferation. Contrary to our expectations, our data showed that the JAK kinase pathway played a repressive role in milk-induced cell proliferation. It has been reported that interleukin-6 (IL-6) inhibits normal intestinal cell proliferation (Booth \& Potten 1995). The JAK kinase pathway plays an important role in the IL-6 signal transduction pathway. In cases of chorioamnionitis, a high concentration of IL-6 is present in the amniotic fluid and fetal plasma. A systemic fetal inflammatory response, as reflected by an elevated fetal plasma concentration of IL- 6 , is an independent risk factor for the occurrence of intestinal diseases with severe neonatal morbidity, such as necrotizing enterocolitis (NEC) (Gomez et al. 1998). The repressive effect of IL-6 on intestinal cell proliferation via the activated JAK kinase pathway may explain this correlation between chorioamnionitis and NEC. Although the details of this milkinduced PTK-dependent pathway remain unknown, such signaling pathways (PTK-dependent but MAP kinase- and PI-3 kinase-independent) are also present in the lung adenocarcinoma cells that are responsible for preventing apoptosis in tumor cells (Wei et al. 2001). It would be interesting to study whether the milk-induced PTK-dependent pathway plays some role in the signal transduction pathway of apoptosis.

Recently, it has been reported that an $\alpha$-lactoalbumin complex from acid-precipitated human milk casein (MAL) induces apoptosis in tumor cells and immature cells, but not in mature differentiated cells (Hakansson et al. 1995). That report showed that the human colonic cancer cell lines, Caco-2 and HT-29, were sensitive to the cytotoxic effects of MAL. FHs 74 Int cells, which we used in our study, were derived from human fetal intestine and show mature epithelial-like characteristics (Wagner et al. 1998). Milk has growth-promoting activity for FHs 74 Int cells as shown in our report and other studies (Ichiba et al. 1992, Wagner et al. 1998, Wagner \& Forsythe 2000) FHs 74 Int cells seem to be resistant to the cytotoxic effect of MAL. We further confirmed that acid-precipitated human milk casein has no cytotoxic effect on this cell line (our unpublished data). Milk may help the neonatal intestinal epithelium towards maturity and away from neoplasia due to the selective sensitivity of tumor cells to MAL.

In this report we showed that the growth-promoting activity of breast milk was contained in the aqueous phase. One of the largest differences between breast milk and artificial formulas is the presence of cellular components in breast milk. Epithelial cells of the mammary gland, macrophages, lymphocytes and neutrophils are present in breast milk and secrete growth modulators into the milk. These cells could survive in the gastrointestinal tract and may also stimulate intestinal cell proliferation by cell-to-cell interactions (Wagner et al. 1996). Fresh whole milk containing living cells may have much higher growth-promoting activity than the aqueous phase of milk used in this study, but fresh whole milk samples did not show any difference from the aqueous phase with respect to growth-promoting activity in our system (our unpublished data). It is thus possible that bioactive substances present in the aqueous phase of milk play a major role in milk-induced intestinal cell proliferation.

While we were preparing this manuscript, Hirai et al. (2002) reported that genistein completely inhibited the growth-promoting activity of milk using the same FHs 74 Int cells. Their results and our data are in accord. Furthermore, in this study, we show for the first time the presence of an unknown signaling pathway, which is different from the previously characterized one induced by EGF. Although our data demonstrated the presence of the unknown signaling pathway, it remains unknown whether this pathway is relevant to the proliferation on the intestinal cells. Further characterization of this signaling pathway will need to show the direct interaction of this pathway to cell proliferation. Our final goal is to identify the bioactive substance in human milk and to characterize the unknown signaling pathway. We are now trying to identify this substance by performing protein purification. As shown in Fig. 2D, this substance is present in the retained fraction of a Microcon Centrifugal Filter Device (YM-100). In the process of protein purification, we confirmed using HPLC that this fraction did not contain any of the low molecular weight substances below $2 \mathrm{kDa}$ in size and that most of the substances were above $20 \mathrm{kDa}$ (our unpublished data). Further examination of the mechanism of the effects of this substance will provide new insights into the regulation of milk-induced intestinal cell proliferation and may help to explain the protective effects of milk on the gastrointestinal tract. This may aid in the development of novel preventive and therapeutic strategies for intestinal diseases of newborn infants such as NEC.

\section{Acknowledgements}

We are grateful to Dr K Nakajima and Dr H Kojima (Osaka City University Medical School) for helpful comments. We thank Dr Y Kaku and Dr M Takase (Morinaga Milk Industry Co., Ltd) for supplying us with acid-precipitated human milk casein. 


\section{References}

Blakesley VA, Scrimgeour A, Esposito D \& Le Roith D 1996 Signaling via the insulin-like growth factor-I receptor: does it differ from insulin receptor signaling? Cytokine Growth Factor Review 7 153-159.

Booth C \& Potten CS 1995 Effects of IL-11 on the growth of intestinal epithelial cells in vitro. Cell Proliferation 28 581-585.

Carpenter G 1980 Epidermal growth factor is a major growth promoting agent in human milk. Science 210 198-199.

Corps AN \& Brown KD 1987 Stimulation of intestinal epithelial cell proliferation in culture by growth factors in human and ruminant mammary secretions. Journal of Endocrinology 113 285-290.

Gomez R, Romero R, Ghezzi F, Yoon BH, Mazor M \& Berry SM 1998 The fetal inflammatory response syndrome. American Journal of Obstetrics and Gynecology 179 194-202.

Grosvenor CE, Picciano MF \& Baumrucker CR 1993 Hormones and growth factors in milk. Endocrine Reviews 14 710-728.

Hakansson A, Zhivotovsky B, Orrenius S, Sabharwal H \& Svanborg C 1995 Apoptosis induced by a human milk protein. PNAS 92 $8064-8068$

Hirai C, Ichiba H, Saito M, Shintaku H, Yamano T \& Kusuda S 2002 Trophic effect of multiple growth factors in amniotic fluid or human milk on cultured human fetal small intestinal cells. Journal of Pediatrics Gastroenterology and Nutrition 34 524-528.

Hirano T 1998 Interleukin 6 and its receptor: ten years later. International Review of Immunology 16 249-284.

Ichiba H, Kusuda S, Itagane Y, Fujita K \& Issiki G 1992 Measurement of growth promoting activity in human milk using a fetal small intestinal cell line. Biology of the Neonate 61 47-53.

Lucas A, Morley R \& Cole TJ 1998 Randomised trial of early diet in preterm babies and later intelligence quotient. British Medical Journal 317 1481-1487.

Lutticken C, Wegenka UM, Yuan J, Buschmann J, Schindler C, Ziemiecki A, Harpur AG, Wilks AF, Yasukawa K, Taga T et al. 1994 Association of transcription factor APRF and protein kinase Jak1 with the interleukin-6 signal transducer gp130. Science 263 89-92.

Moghal N \& Sternberg PW 1999 Multiple positive and negative regulators of signaling by the EGF-receptor. Current Opinion in Cell Biology 11 190-196.

Pimental RA, Julian J, Gendler SJ \& Carson DD 1996 Synthesis and intracellular trafficking of Muc-1 and mucins by polarized mouse uterine epithelial cells. Journal of Biological Chemistry 271 28128-28137.
Provost PR, Blomquist CH, Godin C, Huang XF, Flamand N, Luu-The V, Nadeau D \& Tremblay Y 2000 Androgen formation and metabolism in the pulmonary epithelial cell line A549: expression of 17 beta-hydroxysteroid dehydrogenase type 5 and 3 alpha-hydroxysteroid dehydrogenase type 3. Endocrinology 141 2786-2794.

Read LC, Upton FM, Francis GL, Wallace JC, Dahlenberg GW \& Ballard FJ 1984 Changes in the growth-promoting activity of human milk during lactation. Pediatric Research 18 133-139.

Schanler RJ \& Atkinson SA 1999 Effects of nutrients in human milk on the recipient premature infant. Journal of Mammary Gland Biology and Neoplasia 4 297-307.

Smith-Kirwin SM, O’Connor DM, De Johnston J, Lancey ED, Hassink SG \& Funanage VL 1998 Leptin expression in human mammary epithelial cells and breast milk. Journal of Clinical Endocrinology and Metabolism 83 1810-1813.

Takeda T, Nakajima K, Kojima H \& Hirano T 1994 E1A repression of IL-6-induced gene activation by blocking the assembly of IL-6 response element binding complexes. Journal of Immunology 153 4573-4582.

Wagner CL \& Forsythe DW 2000 Effect of human milk and recombinant EGF, TGFalpha, and IGF-I on small intestinal cell proliferation. Advances in Experimental Medicine and Biology 847 373-374.

Wagner CL, Anderson DM \& Pittard WB 3rd 1996 Special properties of human milk. Clinical Pediatrics 35 283-293.

Wagner CL, Forsythe DW \& Wagner MT 1998 The effect of recombinant TGF $\alpha$, human milk, and human milk macrophage media on gut epithelial proliferation is decreased in the presence of a neutralizing TGF $\alpha$ antibody. Biology of the Neonate 74 363-371.

Wei L, Yang Y \& Yu Q 2001 Tyrosine kinase dependent, phosphatidylinositol-3' kinase, and mitogen-activated protein kinase-independent signaling pathways prevent lung adenocarcinoma cells from anoikis. Cancer Research 61 2439-2444.

Zhong Z, Wen Z \& Darnell JE Jr 1994 Stat3: a STAT family member activated by tyrosine phosphorylation in response to epidermal growth factor and interleukin-6. Science 264 95-98.

Zong CS, Chan J, Levy DE, Horvath C, Sadowski HB \& Wang LH 2000 Mechanism of STAT3 activation by insulin-like growth factor I receptor. Journal of Biological Chemistry 275 15099-15105.

Received 25 February 2004

Accepted 3 March 2004 